\title{
Erratum: Prospective constraints on the primordial black hole abundance from the stochastic gravitational-wave backgrounds produced by coalescing events and curvature perturbations [Phys. Rev. D 99, 103531 (2019)]
}

\author{
Sai Wang, Takahiro Terada๑, and Kazunori Kohri
}

(Q) (Received 3 March 2020; accepted 5 March 2020; published 16 March 2020)

DOI: 10.1103/PhysRevD.101.069901

Though the formula in Eq. (11) in our paper is correct, we unfortunately missed a factor of $2 \pi$ in Fig. 4, implying that we used a relation $\nu=k$ instead of $\nu=k / 2 \pi$ in our evaluation. Here, $\nu$ and $k$ denote the frequency and wave number of gravitational waves, respectively. If we take this factor into account in Fig. 4, the energy-density spectrum of the induced gravitational-wave background should be globally shifted to the left-hand side by a factor of $2 \pi$. In this way, SKA would have intersections with all the energy-density spectra of the induced gravitational-wave background in Fig. 5, while LISA would not have intersections with any spectrum. Therefore, by considering the induced gravitational-wave background, SKA is expected to test the possibility of primordial black holes being (a part of) cold dark matter over the whole mass range in Figs. 6 and 7, while LISA is expected not to improve the existing constraints on the abundance of primordial black holes.

We should stress, however, that a more realistic power spectrum of curvature perturbations will lead to qualitatively similar figures to those before this erratum. This is partially because we assumed a monochromatic power spectrum for simplicity, which is not generic in the sense that the resultant gravitational-wave spectrum has a broad $\nu^{2}$ tail in the small $\nu$ part, while any spectra with a finite width lead to a steeper $\nu^{3}$ scaling in the sufficiently small $\nu$ region (see Footnote 2 in our paper). It is also because we cut the right part of the second-order gravitational-wave spectrum which has a sharp peak. If we change these assumptions, the SKA constraints become weaker, and the LISA constraints become stronger. We leave such analyses for more realistic cases for future work. 